\title{
DISSEMINATED ASPERGILLOSIS IN AN AUTOPSY CASE OF YOUNG MALE
}

Swarnagowri B. N ${ }^{1}$, Shubha Sangeetha ${ }^{2}$

\section{HOW TO CITE THIS ARTICLE:}

Swarnagowri B. N, Shubha Sangeetha. "Disseminated Aspergillosis in an Autopsy Case of Young Male". Journal of Evolution of Medical and Dental Sciences 2014; Vol. 3, Issue 23, June 09; Page: 6382-6385,

DOI: $10.14260 /$ jemds/2014/2751

ABSTRACT: Aspergillus is a ubiquitous mold that causes allergies (allergic bronchopulmonary aspergillosis) in otherwise healthy people and serious sinusitis, pneumonia and invasive disease in immuno-compromised individuals. The major conditions that predispose to Aspergillus infection are neutropenia and corticosteroids. Aspergillus fumigatus is the most common species to cause disease, and it produces severe invasive infections in immunocompromised individuals. We get around 20 autopsy specimens per month in our institution. This case was interesting because of the rarity associated with the immunocompromised young male of $27 \mathrm{yrs}$.

KEYWORDS: Lung, Aspergillus infection, invasive aspergillosis, immunosuppression.

INTRODUCTION: The fungus Aspergillus was baptized by the priest and botanist Micheli in 1729 since it bore resemblance to the perforated globe used for sprinkling holy water. ${ }^{2}$ Aspergillus is a common cause of invasive mycosis, especially in immunocompromised or immunosuppressed individuals. The incidence has been found to be $0.19 \% .{ }^{1}$ Aspergillus fumigatus, a ubiquitous organism that is capable of living under extensive environmental stress. It is estimated that most humans inhale thousands of Aspergillus spores daily, but they do not impact on most people's health due to effective immune responses.

Aspergillus species are transmitted by airborne conidia, and the lung is the major portal of entry. The small size of A. fumigatus spores, approximately 2 to $3 \mu \mathrm{m}$, enables them to reach alveoli. Conidia germinate into hyphae, which then invade tissues. Neutrophils and macrophages are the major host defenses against Aspergillus. Alveolar macrophages ingest and kill the conidia, while neutrophils produce reactive oxygen intermediates that kill hyphae. Invasive aspergillosis is highly associated with neutropenia and impaired neutrophil defences. ${ }^{2}$ Invasive aspergillosis is an opportunistic infection that is confined to immunosuppressed hosts. The primary lesions are usually in the lung, but widespread haematogenous dissemination with involvement of the heart valves and brain is common.

The pulmonary lesions take the form of necrotizing pneumonia with sharply delineated, rounded, gray foci and hemorrhagic borders.They are often referred to as target lesions.

Aspergillus forms fruiting bodies (usually in lung cavities) and septate filaments, 5 to $10 \mu \mathrm{m}$ thick, branching at acute angles (40 degrees). Aspergillus has a tendency to invade blood vessels; therefore, areas of haemorrhage and infarction are usually superimposed on the necrotizing, inflammatory tissue reactions. ${ }^{3}$

CASE REPORT: We had an autopsy case of $27 \mathrm{yr}$ male with h/o sudden death. On gross examination, all the organs were congested and no other specific lesion was noted. Microscopy revealed the fungal filaments and colonies in the alveoli of the lungs, in liver, spleen, kidney showing these filaments in the interstitium with granulomatous lesion and also left circumflex artery showing these fungal balls 


\section{CASE REPORT}

in the thrombus within the lumen.[Figs.1, 2, 3, \&4].So a diagnosis of disseminated Aspergillosis was made, based on the histopathological findings. The lab investigation details of the deceased could not be obtained.

Only we could gather the information was he was in a high risk job and immunocompromised. Periodic acid Schiff and silver (Gomori Methanamine Silver) stains were done, which showed positivity for fungal filaments. These were identified as regular slender, septate hyphae that branch dichotomously at an angle of 45 degrees.

DISCUSSION: Aspergillus produces several virulence factors, including adhesins, antioxidants, enzymes, and toxins 4 . Conidia can bind to fibrinogen, laminin, complement, fibronectin, collagen, albumin, and surfactant proteins, but receptor-ligand interactions are not well defined. Aspergillus produces several antioxidant defenses, including melanin pigment, mannitol, catalases and superoxide dismutases. This fungus also produces phospholipases, proteases and toxins, but their roles in pathogenicity are not yet clear.

Restrictocin and mitogillin are ribotoxins that inhibit host-cell protein synthesis by degrading mRNAs. The carcinogen aflatoxin is made by Aspergillus species growing on the surface of peanuts and may be a cause of liver cancer in Africa. ${ }^{5}$ People with deficient immune systems- such as patients undergoing hematopoietic stem cell transplantation, chemotherapy for leukaemia, or AIDS-are at risk of more disseminated disease. Acute invasive aspergillosis occurs when the immune system fails to prevent Aspergillus spores from entering the bloodstream via the lungs. Without the body mounting an effective immune response, fungal filaments are free to disseminate throughout the body and can infect major organs such as the heart and kidneys. Rapidly invasive Aspergillus infection in the lungs often causes cough, fever, chest pain and difficulty in breathing. Poorly controlled aspergillosis can disseminate through the blood stream to cause widespread organ damage. Symptoms include fever, chills, shock, delirium, seizures and blood clots. The person may develop kidney failure, liver failure and breathing difficulties. Death can occur quickly.

CONCLUSION: Invasive aspergillosis is a potentially fatal complication of immunosuppression. Aspergillosis occurs in the form of chronic pulmonary aspergillosis (CPA), aspergilloma or allergic bronchopulmonary aspergillosis. Prevention of Aspergillosis involves a reduction of mold exposure via environmental infection-control ${ }^{7}$

Risk factors are weak immune system, AIDS, low white blood cell levels. Having a very low level of these cells (neutropenia) due to chemotherapy, an organ transplant or leukaemia makes it more susceptible to invasive aspergillosis. So does having chronic granulomatous disease,an inherited disorder that affects immune system cells, lung cavities that have been damaged by radiation to the lung or by lung diseases such as tuberculosis or sarcoidosis.

People with asthma and cystic fibrosis are more likely to have an allergic response to aspergillus mold. Ankylosing spondylitis. This is an uncommon rheumatologic lung disease that affects primarily the spine. People with this disorder are more likely to develop aspergillomas, particularly if they are male smokers. Long-term use of corticosteroids may increase the risk of opportunistic infections, depending on the underlying disease being treated and what other drugs are 
being used. Aspergillus mold is found on many hospital surfaces-bedrails, plants, surgical instruments, air conditioning ducts and insulation.

Depending on the type of infection, aspergillosis can cause a variety of serious complications:

- Bone destruction and spread of infection.

- Bleeding in the lungs.

- Systemic infection. The most serious complication of invasive aspergillosis is the spread of the infection to other parts of the body, especially brain, heart and kidneys. Invasive aspergillosis spreads rapidly and is often fatal in spite of early treatment.

The investigations include the following:

- Imaging test. A chest X-ray or computerized tomography (CT) scan can usually reveal a fungal mass (aspergilloma), as well as characteristic signs of invasive and allergic bronchopulmonary aspergillosis.

- Sputum test.

- Tissue and blood tests. Diagnosing allergic bronchopulmonary aspergillosis usually requires skin and blood tests. For the skin test, a small amount of aspergillus antigen is injected into the skin of the forearm. If the blood has antibodies to the mold, induration will develop at the injection site. Blood tests reveal high levels of certain antibodies, indicating an allergic response.

- Biopsy. Examining a sample of tissue from lungs or sinuses is usually necessary to confirm a diagnosis of invasive aspergillosis.

\section{REFERENCES:}

1. Invasive pulmonary aspergillosis: A study of 39 cases at autopsy. Vaideeswar P1, Prasad S, Deshpande JR, Pandit SP. J Postgrad Med March 2004; Vol. 50 Issue 1.

2. Robinson LA, Reed EC, Galbraith TA, Alonso A, Moulton AL, Fleming WH. Pulmonary resection of invasive Aspergillus infections in immunocompromised patients. J Thorac Cardiovasc Surg 1995; 109:1182-97.

3. Robbins and Cotran Pathologic basis of disease $8^{\text {th }}$ edition.

4. Tuder RM, et al: Pathology of pulmonary hypertension. Clin Chest Med 2007; 28:23.

5. Fuster V, et al: Primary pulmonary hypertension: natural history and the importance of thrombosis. Circulation 1984; 70:580.

6. Kradin RL, Mark EJ (April 2008). "The pathology of pulmonary disorders due to Aspergillus spp". Arch. Pathol. Lab. Med. 132 (4): 606-14.

7. Cornely, Oliver A.; Maertens, Johan; et al (2007). "Posaconazole vs. Fluconazole or Itraconazole Prophylaxis in Patients with Neutropenia". New England Journal of Medicine 356 (4): 348-359. 


\section{CASE REPORT}
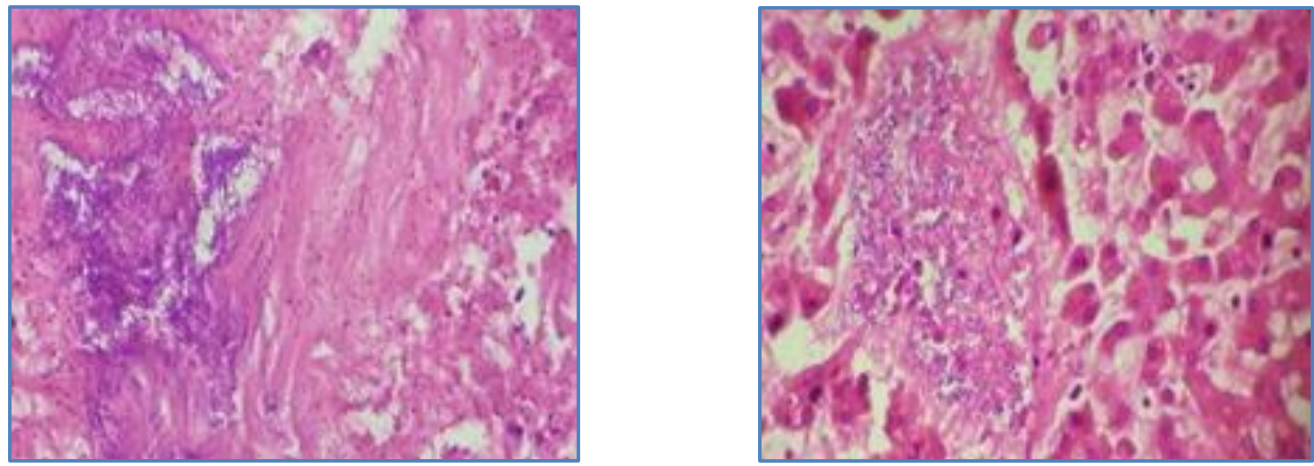

Fig. 1 \& 2: (10x) showing Aspergillus filaments in lung and liver
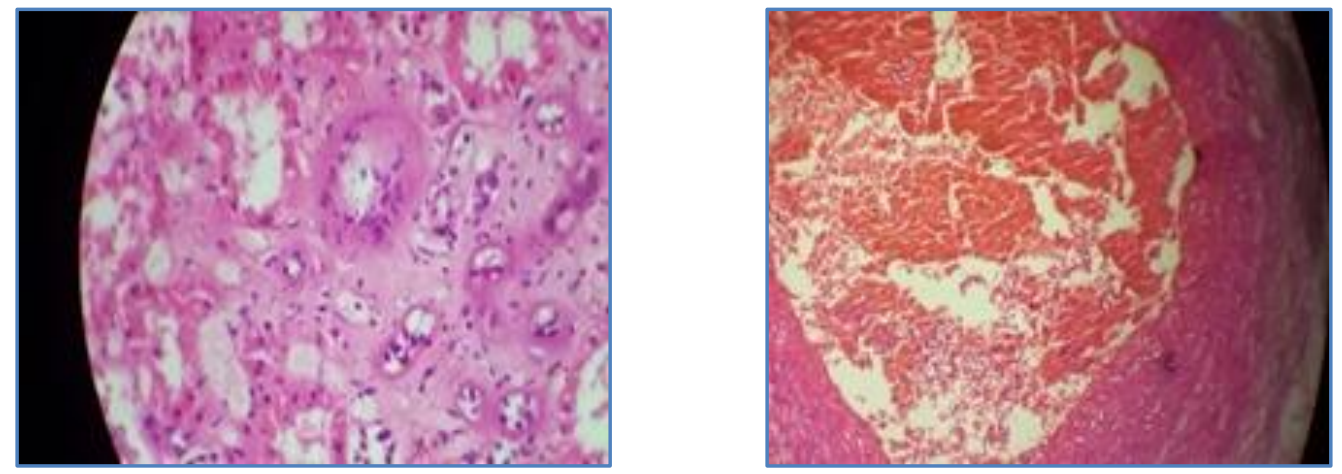

Fig. 3 \& 4: (10x) showing granulomas in the kidney and Aspergillus filaments in a thrombus occluding the lumen of left circumflex artery

\section{AUTHORS:}

1. Swarnagowri B. N.

2. Shubha Sangeetha

\section{PARTICULARS OF CONTRIBUTORS:}

1. Professor, Department of Pathology, Dr. B. R. Ambedkar Medical College, Bangalore.

2. Assistant Professor, Department of Pathology, Dr. B. R. Ambedkar Medical College, Bangalore.
NAME ADDRESS EMAIL ID OF THE

\section{CORRESPONDING AUTHOR:}

Dr. Swarnagowari B. N,

No. 21, $6^{\text {th }}$ Cross,

Vasanthnagar,

Bangalore-560001.

E-mail: swarnagowri@ymail.com

Date of Submission: 26/05/2014.

Date of Peer Review: 27/05/2014.

Date of Acceptance: 31/05/2014.

Date of Publishing: 05/06/2014. 